\title{
REVIEW
}

\section{Chemometric Approaches in Questioned Documents}

\author{
Roberta Petry Gorziza1* $ه$, Marina González ${ }^{\text {* }}$ iD, Carina Maria Bello de Carvalho ${ }^{\text {iD }}$ \\ Rafael Scorsatto Ortiz ${ }^{2,3}$ iD, Marco Flores Ferrão ${ }^{4,5}$ iD, Renata Pereira Limberger ${ }^{1,3}$ iD \\ ${ }^{1}$ Departamento de Farmácia, Universidade Federal do Rio Grande do Sul, Av. Ipiranga, 2752, Azenha, \\ 90610-000, Porto Alegre, RS, Brazil \\ ${ }^{2}$ Polícia Federal, Divisão Técnico-Científica do Rio Grande do Sul, Av. Ipiranga, 1365, Azenha, \\ 90610-093, Porto Alegre, RS, Brazil \\ ${ }^{3}$ Instituto Nacional de Ciência e Tecnologia Forense (INCT Forense), Porto Alegre, RS, Brazil \\ ${ }^{4}$ Departamento de Química, Universidade Federal do Rio Grande do Sul, Porto Alegre, RS Brazil \\ 5Instituto Nacional de Ciência e Tecnologia em Bioanalítica (INCT Bioanalítica), Campinas, SP, Brazil \\ *These authors contributed equally.
}

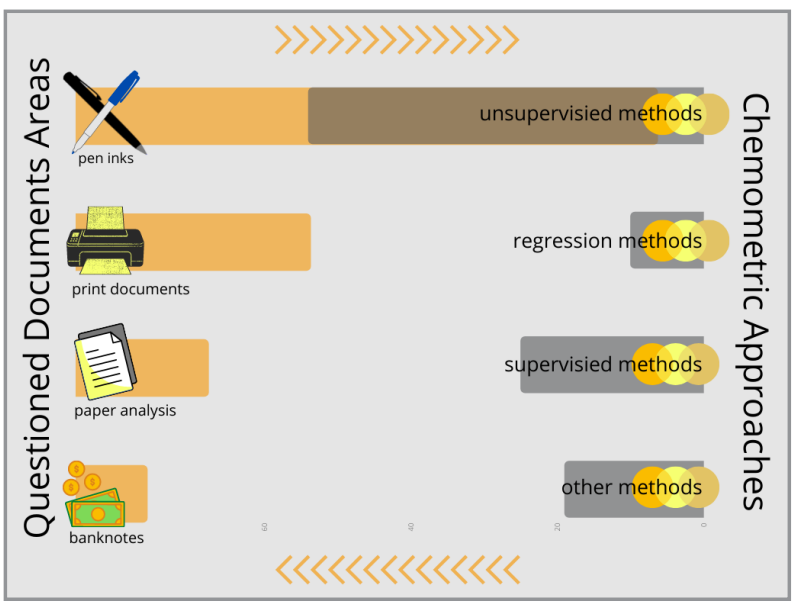

Questioned documents comprehend analysis of identity theft, forged signatures or texts, documents alterations and falsification of security documents or banknotes. Questions involving inks or paper require chemical analysis, and multivariate analysis or chemometrics has been an emerging tool for data evaluation and interpretation after instrumental data collection in this area. The goal of this study is to identify previous articles that applied multivariate analysis within questioned documents for forensic purposes. The search for articles was performed in four databases (Google Scholar, Science Direct, Pubmed and Scopus). Sixty studies, published in the last ten years, were selected. Thirtyfour articles described pen inks analysis; fourteen articles comprehended printed documents studies; eight articles evaluated paper analysis, and four articles included banknotes analysis. Spectroscopy, mass spectrometry, chromatography, thermo gravimetric analysis and multivariate image analysis were the analytical methods applied to collect chemical data. Chemometrics methods included mainly unsupervised pattern recognition techniques, regression methods, and supervised pattern recognition techniques, amongst other methods. This review summarized and discussed multivariate analysis techniques applied in different questioned documents sub-areas, highlighting the importance of this knowledge for forensic analysts. In addition, it shows new research topics such as different printing and pen inks, papers and security documents analysis herein not included.

Keywords: questioned documents, inks, paper, chemometrics, multivariate analysis.

Cite: Gorziza, R. P.; González, M.; de Carvalho, C. M. B.; Ortiz, R. S.; Ferrão, M. F.; Limberger, R. P. Chemometric Approaches in Questioned Documents: A Review. Braz. J. Anal. Chem., 2022, 9 (34), pp 35-51. doi: http://dx.doi.org/10.30744/brjac.2179-3425. RV-32-2021 


\section{INTRODUCTION}

Questioned documents is an important area of Forensic Sciences, comprehending document fraud analysis such as identity theft, forged signatures or texts, documents alterations and falsification of security documents or banknotes. While physical analysis is widely applied in questioned documents, many situations demand the chemical analysis of inks and support paper [1-3].

Writing and printing inks are formulas composed by pigments or dyes, resins, solvents, driers and drying oils, extenders and additives, such as surfactants, conductive salts, biocides, composite carriers, or adhesion promoters [4]. Writing inks comprises different types of pens, such as ballpoint pens (oil-based inks composed of dyes or pigments and organic solvents), gel pens (water-based inks composed mainly of pigments), and rollerball, fountain and felt-tip pens (water-based inks composed of dyes and ethylene glycol) [4]. The most prevalent printing inks are inkjets and toners. Inkjet inks can be solvent-based, waterbased, UV curable, and phase-change, depending on the printer instrument; toner inks are dry powders or liquid-dispersed powders, mainly constituted of pigments and resins [4].

The paper production from wood demands pulping and bleaching process, using chemical agents such as sodium sulfide $\left(\mathrm{Na}_{2} \mathrm{~S}\right)$, sodium hydroxide $(\mathrm{NaOH})$, chlorine monoxide $\left(\mathrm{Cl}_{2} \mathrm{O}\right)$, calcium carbonate $\left(\mathrm{CaCO}_{3}\right)$, ozone or oxygen, with metallic oxides. Thus, the final paper products contain cellulose and particular compounds, specific of each production set [1].

In questioned documents forensic examination, inks and papers characterization and transformation are often required for differentiation and age estimation, respectively [2]. In addition, counterfeit security documents and banknotes must be differentiated from the authentic ones. In this regard, numerous different analytical methods have been studied [1,3,5-8]. Many analytical methods, for the chemical analysis of inks and paper, produce a huge amount of data outputs, demanding an efficient and accurate method for results interpretation. Multivariate analysis in chemistry, or chemometrics, are statistical approaches for chemical data analysis $[9,10]$. Additionally, it can be used for planning and simulations of chemical experiments $[9,10]$. Chemometrics offer trustworthy tests for classification, discrimination or models development for different chemical samples datasets [11]. Thus, chemometrics are emerging tools in Forensic Sciences, as mathematical and statistical tools are potential methods to enrich and to correlate forensic analytical data in many areas besides questioned documents (biological, physical and chemical sciences, toxicology, ballistic, anthropology) [11]. While many analytical methods are applied for inks and paper examination, chemometrics can improve results interpretation and data presentation towards a forensic document investigation. Chemometrics increases the data analysis objectivity in a comparison study, and it is a powerful tool to perform databases research. Hence, chemometrics is a growing trend in questioned documents data analysis, and this knowledge is important for forensics analysts [3].

The aim of this work is to review questioned documents topics which were studied by multivariate analysis or chemometrics approaches, highlighting its importance in the field. This review summarizes research studies that applied chemometrics in questioned documents analysis, and if offers a brief explanation of the most applied chemometrics techniques. It also identifies the most covered research topics in questioned documents, and the analytical methodologies performed prior to chemometrics analysis. Therefore, this review detects areas and methodologies that could be explored in further research.

\section{MATERIALS AND METHODS}

Figure 1 summarizes the articles search method. The search for articles was performed in November and December 2020, on four different databases, using the descriptor "multivariate analysis" or "chemometrics" and questioned documents. The search was performed in the last ten years (2010-2020). The initial search from Google Scholar (1610 results/55 articles selected), Science Direct (190 results/16 articles selected), Pubmed (70 results/10 articles selected) and Scopus (21 results/18 articles selected) retrieved 62 articles, when analyzing titles and abstracts. The inclusion criteria consisted of any study within the questioned documents area, as long as it applied multivariate analysis or chemometric data analysis. Questioned documents articles that did not describe these techniques were excluded of the search. Only original 
articles written in English or Portuguese were included. After the exclusion of repeated and review papers, 50 articles were selected and organized by area (pen inks, printed documents, paper and banknotes analysis).

In order to find as many articles as possible, complementary searches were performed on Google Scholar. For these searches, the term "questioned documents" was substituted from the descriptor "multivariate analysis" or "chemometrics" and questioned documents, by using specific terms related to each area, one at a time: "pen inks", "printed", "prints", "printing", "inkjet", "toners", "stamps", "packages", "packaging", "banknotes", and "paper analysis". Finally, references from questioned documents review papers $[1,3]$ were also analyzed, to retrieve any missing article applying chemometric approaches in the last ten years.

A total of 60 studies applying multivariate analysis/chemometrics in the questioned documents field were selected.

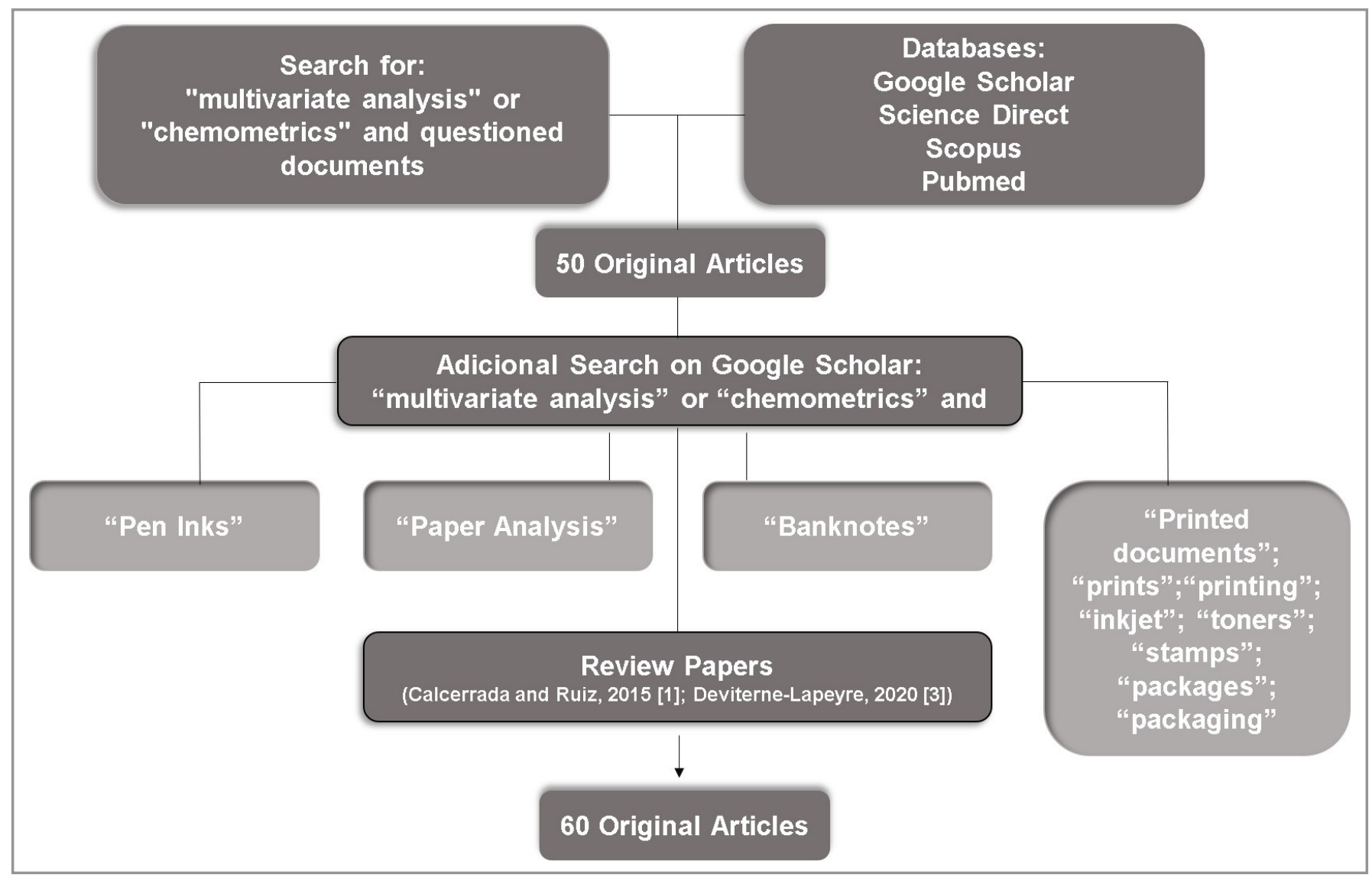

Figure 1. Articles search strategy. 


\section{RESULTS AND DISCUSSION}

Four major questioned documents areas were contemplated with multivariate analysis/chemometrics studies. Figure 2 shows the articles distribution among the different areas.

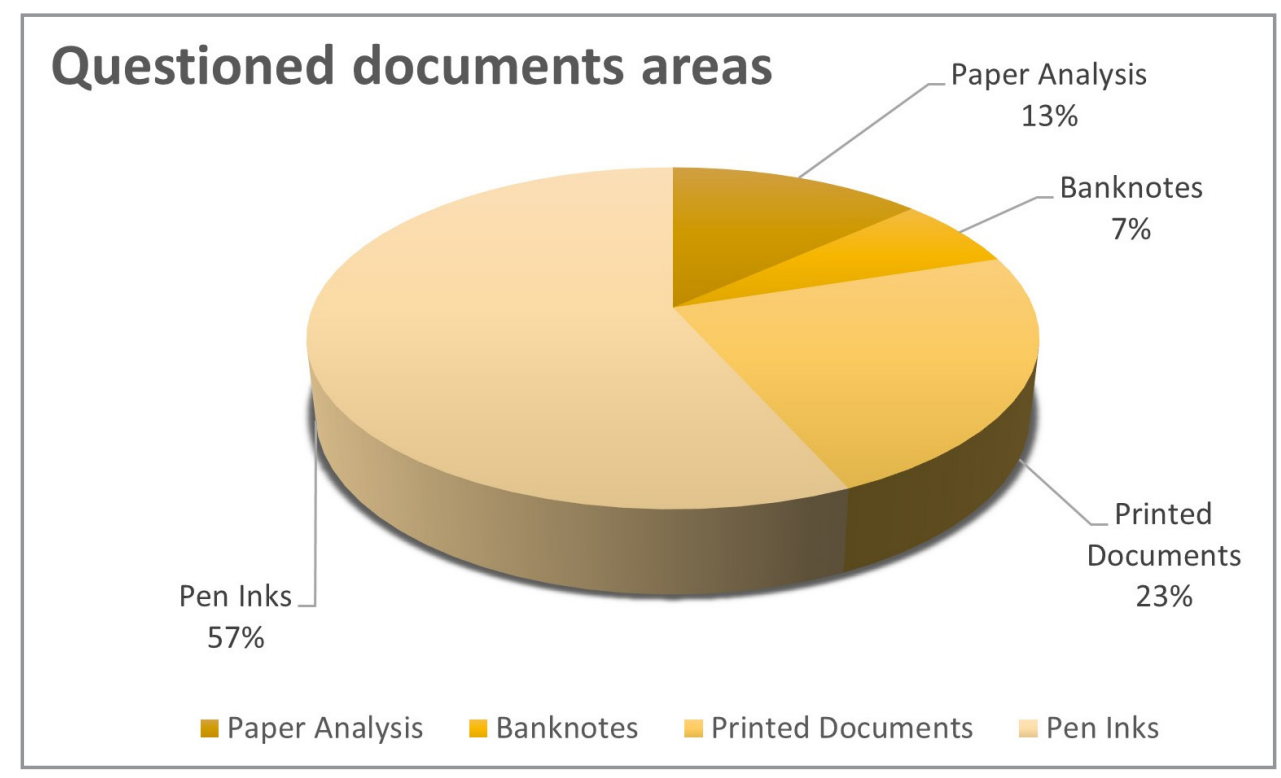

Figure 2. Multivariate analysis/chemometric articles distribution among different questioned documents areas.

Pen inks analysis comprehended 34 articles (57\% from total studies). Table I summarizes these studies. Among these, 7 articles utilized mainly spectroscopy techniques to study ballpoint pen ink dating; 16 articles utilized spectroscopy, mass spectrometry and multivariate image analysis to study ballpoint pen inks differentiation; 4 studies applied spectroscopy techniques for marker pen inks, gel pen inks and fiber tip pen inks differentiation, and 4 studies evaluated different types - or classes - of pens, using multivariate image analysis, mass spectrometry and spectroscopy methods. Thus, 4 studies analyzed pen ink crossing lines, between ballpoint pens, between gel pens or between ballpoint and gel pens, using spectroscopy and mass spectrometry techniques.

Table II shows 14 studies applied on printed documents and printer inks, representing $23 \%$ from total studies. These documents comprised food packages, pharmaceutical packages, and stamps. Printed inks included toners, inkjets, off set and intaglio inks. Also, crossing lines studies involving printing inks, papers and pen inks were conducted. Although spectroscopic methods were the most applied techniques in this area, mass spectrometry and multivariate image analysis were also studied.

Table III shows 8 studies applied on paper analysis, which represents $13 \%$ from total studies. Among these studies, 3 articles aimed to characterize or to discriminate different types of paper, while 5 articles focused on paper age estimation. Spectroscopy methods were the most prevalent techniques applied for paper analysis, along with analytical pyrolysis combined with gas chromatography/mass spectrometry and thermo gravimetric analysis.

The last questioned documents area comprehended banknotes analysis. Table IV shows 4 studies ( $7 \%$ of total studies) of banknotes - mainly Brazilian - classification and counterfeit/authentic bills differentiation, applying spectroscopy techniques, spectrometry, and multivariate image analysis. 
Table I. Studies concerning pen inks analysis and multivariate analysis/chemometrics approaches

\begin{tabular}{|c|c|c|c|c|c|}
\hline Main Objectives & Samples & $\begin{array}{l}\text { Analytical } \\
\text { Method }\end{array}$ & $\begin{array}{l}\text { Multivariate } \\
\text { Analysis }\end{array}$ & Software & Reference \\
\hline Ballpoint pen ink dating & $\begin{array}{l}11 \text { blue ballpoint } \\
\text { pens from } 6 \text { brands }\end{array}$ & Vis-MSP $^{1}$ & $\begin{array}{l}\mathrm{PCA}^{20}, \mathrm{HCA}^{21} \\
\text { and } \mathrm{OPLS}^{22}\end{array}$ & SIMCA 15.0.2 & $\begin{array}{l}\text { Ortiz-Herrero et } \\
\text { al. [12] }\end{array}$ \\
\hline Ballpoint pen ink dating & $\begin{array}{l}505 \text { ballpoint pens } \\
\text { inks ( } 384 \text { blue and } \\
121 \text { black) }\end{array}$ & DI-MS² & $\mathrm{ULT}^{23}$ & Analyst ${ }^{\circledR}$ TF 1.6 & Costa et al. [13] \\
\hline Ballpoint pen ink dating & $\begin{array}{l}37 \text { blue and } 27 \text { black } \\
\text { ballpoint pens }\end{array}$ & ATR-FTIR ${ }^{3}$ & $\begin{array}{c}\mathrm{PCA}^{20} \text { and } \\
\mathrm{HCA}^{21}\end{array}$ & Chemostat $^{\circledR}$ & $\begin{array}{c}\text { Bello de Carvalho } \\
\text { et al. [14] }\end{array}$ \\
\hline Ballpoint pen ink dating & $\begin{array}{l}4 \text { black and } 1 \text { blue } \\
\text { ballpoint pens }\end{array}$ & UV-Vis-NIR ${ }^{4}$ & $\mathrm{PLS}^{24}$ & SIMCA 13.0 & $\begin{array}{l}\text { Ortiz-Herrero et } \\
\text { al. [15] }\end{array}$ \\
\hline Ballpoint pen ink dating & Blue ballpoint pen & UV-Vis ${ }^{5}$ & $\begin{array}{l}\mathrm{PCA}^{20} \text { and } \\
\mathrm{MLR}^{25}\end{array}$ & SPSS $^{\circledR}$ & $\begin{array}{c}\text { Sharma \& Kumar. } \\
{[16]}\end{array}$ \\
\hline Ball tip pen inks dating & 35 blue ball tips pens & UV-Vis ${ }^{5}$ & $\begin{array}{l}\mathrm{PCA}^{20}, \mathrm{LDA}^{26} \\
\text { and } \mathrm{PLSR}^{27}\end{array}$ & $\begin{array}{c}\text { Unscrambler X } \\
10.4\end{array}$ & Sauzier et al. [17] \\
\hline $\begin{array}{l}\text { Ballpoint pen inks } \\
\text { characterization and } \\
\text { dating }\end{array}$ & $\begin{array}{l}10 \text { blue ballpoint } \\
\text { pens }\end{array}$ & $\begin{array}{c}\text { UV-Vis }{ }^{5}, I^{6} \text { and } \\
\text { HPTLC }^{7}\end{array}$ & $\mathrm{PCA}^{20}$ & Microsoft Excel & Senior et al. [18] \\
\hline $\begin{array}{l}\text { To differentiate } \\
\text { ballpoint pen inks }\end{array}$ & $\begin{array}{l}12 \text { blue ballpoint } \\
\text { pens from } 9 \text { brands }\end{array}$ & $\mathrm{MIA}^{8} /$ Smartphone & $\begin{array}{l}\mathrm{PCA}^{20}, \mathrm{HCA}^{21} \\
\text { and PLS-DA }\end{array}$ & PhotometrixPRO ${ }^{\circledR}$ & Gorziza et al. [19] \\
\hline $\begin{array}{l}\text { To differentiate } \\
\text { ballpoint pen inks }\end{array}$ & $\begin{array}{l}7 \text { different blue } \\
\text { ballpoint pens }\end{array}$ & Raman Imaging & MF-ICA 29 & MATLAB ${ }^{\circledR}$ & Teixeira et al. [20] \\
\hline $\begin{array}{l}\text { To differentiate } \\
\text { ballpoint pen inks }\end{array}$ & $\begin{array}{l}33 \text { blue and } 36 \text { black } \\
\text { ballpoint pens }\end{array}$ & Orbitrap $\mathrm{MS}^{9}$ & $\begin{array}{c}\mathrm{PCA}^{20} \text { and } \\
\mathrm{HCA}^{21}\end{array}$ & Chemostat $^{\circledR}$ & $\begin{array}{c}\text { Bello de Carvalho } \\
\text { et al. [21] }\end{array}$ \\
\hline $\begin{array}{l}\text { To differentiate } \\
\text { ballpoint pen inks }\end{array}$ & $\begin{array}{l}11 \text { unbranded black } \\
\text { ballpoint pens }\end{array}$ & $\mathrm{FTIR}^{10}$ & $\begin{array}{c}\mathrm{PCA}^{20} \text { and } \\
\mathrm{HCA}^{21}\end{array}$ & Minitab ${ }^{\circledR}$ & Kamil et al. [22] \\
\hline $\begin{array}{l}\text { To differentiate } \\
\text { ballpoint pen inks }\end{array}$ & $\begin{array}{l}36 \text { black ballpoint } \\
\text { pens from } 6 \text { brands }\end{array}$ & PS-MS 11 & $\mathrm{PLS}^{24}$ & MATLAB $^{\circledR}$ & Amador et al. [23] \\
\hline $\begin{array}{l}\text { To differentiate } \\
\text { ballpoint pen inks }\end{array}$ & $\begin{array}{l}57 \text { blue ballpoint } \\
\text { pens }\end{array}$ & $\begin{array}{c}\text { ATR-FTIR }^{3} \text { and } \\
\text { HPTLC }^{7}\end{array}$ & $\mathrm{PCA}^{20}$ & SPSS-20 ${ }^{\circledR}$ & $\begin{array}{l}\text { Sharma and } \\
\text { Kumar. [24] }\end{array}$ \\
\hline $\begin{array}{l}\text { To differentiate } \\
\text { ballpoint pen inks }\end{array}$ & $\begin{array}{l}30 \text { ballpoint pens } \\
\text { (blue and red) from } 5 \\
\text { brands }\end{array}$ & $\begin{array}{c}\text { Raman } \\
\text { Spectroscopy }\end{array}$ & $\mathrm{PCA}^{20}$ & Minitab $^{\circledR}$ & Asri et al. [25] \\
\hline $\begin{array}{l}\text { To differentiate } \\
\text { ballpoint pen inks }\end{array}$ & $\begin{array}{l}24 \text { black ballpoint } \\
\text { pens of } 6 \text { different } \\
\text { brands }\end{array}$ & micro-ATR-FTIR ${ }^{3}$ & $\mathrm{PCA}^{20}$ & SPSS-15 ${ }^{\circledR}$ & Lee et al. [26] \\
\hline $\begin{array}{l}\text { To differentiate } \\
\text { ballpoint pen inks }\end{array}$ & $\begin{array}{l}155 \text { black ballpoint } \\
\text { pens of } 9 \text { different } \\
\text { brands }\end{array}$ & micro-ATR-FTIR ${ }^{3}$ & $\mathrm{PCA}^{20}$ & SPSS $-15^{\circledR}$ & Lee et al. [27] \\
\hline $\begin{array}{l}\text { To differentiate } \\
\text { ballpoint pen inks }\end{array}$ & $\begin{array}{l}57 \text { blue ballpoint } \\
\text { pens }\end{array}$ & UV-Vis-NIR ${ }^{4}$ & $\mathrm{PCA}^{20}$ & SPSS-20 ${ }^{\circledR}$ & $\begin{array}{l}\text { Kumar and } \\
\text { Sharma [28] }\end{array}$ \\
\hline $\begin{array}{l}\text { To differentiate } \\
\text { ballpoint pen inks }\end{array}$ & $\begin{array}{l}24 \text { blue ballpoint } \\
\text { pens from } 6 \text { brands }\end{array}$ & $\mathrm{FTIR}^{10}$ & $\begin{array}{c}\mathrm{PCA}^{20} \text { and } \\
\mathrm{HCA}^{21}\end{array}$ & XLSTAT 2011 & Halim et al. [29] \\
\hline $\begin{array}{l}\text { To differentiate } \\
\text { ballpoint pen inks }\end{array}$ & $\begin{array}{l}14 \text { pen ink classes of } \\
\text { blue ballpoint pens }\end{array}$ & $\begin{array}{c}\text { Raman } \\
\text { Spectroscopy }\end{array}$ & $\begin{array}{l}\mathrm{PCA}^{20}, \mathrm{HCA}^{21} \\
\text { and PLS-DA }\end{array}$ & Not informed & Borba et al. [30] \\
\hline $\begin{array}{l}\text { To differentiate } \\
\text { ballpoint pen inks }\end{array}$ & $\begin{array}{l}21 \text { blue ballpoint } \\
\text { pens from } 10 \text { brands }\end{array}$ & LA-ICP-MS ${ }^{12}$ & MANOVA $^{30}$ & SPSS $^{\circledR}$ & Alamilla et al. [31] \\
\hline
\end{tabular}


Table I. Studies concerning pen inks analysis and multivariate analysis/chemometrics approaches (Continuation)

\begin{tabular}{|c|c|c|c|c|c|}
\hline Main Objectives & Samples & $\begin{array}{l}\text { Analytical } \\
\text { Method }\end{array}$ & $\begin{array}{c}\text { Multivariate } \\
\text { Analysis }\end{array}$ & Software & Reference \\
\hline $\begin{array}{l}\text { To differentiate } \\
\text { model variation of } \\
\text { Papermate }{ }^{\circledR} \text { pens }\end{array}$ & $\begin{array}{l}37 \text { black ballpoint } \\
\text { pens }\end{array}$ & micro-ATR-FTIR ${ }^{3}$ & $\mathrm{PCA}^{20}$ & SPSS $-12^{\circledR}$ & Lee et al. [32] \\
\hline $\begin{array}{l}\text { To differentiate marker } \\
\text { pen inks }\end{array}$ & 24 markers pen inks & $\begin{array}{l}\text { UV-Vis }{ }^{5} \text { and UV- } \\
\text { NIR }^{13}\end{array}$ & $\begin{array}{c}\mathrm{PCA}^{20} \text { and } \\
\mathrm{DA}^{31}\end{array}$ & SPSS-16 ${ }^{\circledR}$ & Sharma et al. [33] \\
\hline $\begin{array}{l}\text { To differentiate gel } \\
\text { pens }\end{array}$ & $\begin{array}{l}45 \text { gel pen inks (blue, } \\
\text { red and black) from } 5 \\
\text { brands }\end{array}$ & $\mathrm{HSI}^{14}$ & $\mathrm{PCA}^{20}$ & Minitab ${ }^{\circledR}$ & Asri et al. [34] \\
\hline $\begin{array}{l}\text { To differentiate gel } \\
\text { pens }\end{array}$ & 10 gel pen black inks & LIBS $^{15}$ & $\mathrm{PCA}^{20}$ & $\mathrm{~N} / \mathrm{l}$ & $\begin{array}{l}\text { Ballah and } \\
\text { Nassef [35] }\end{array}$ \\
\hline $\begin{array}{l}\text { To differentiate gel } \\
\text { pens }\end{array}$ & 45 black gel pen inks & $\mathrm{HSI}^{14}$ & $\begin{array}{l}\mathrm{PCA}^{20}, \mathrm{HCA}^{21} \\
\text { and } \mathrm{SAM}^{32}\end{array}$ & Statistica & $\begin{array}{c}\text { Chlebda et al. } \\
{[36]}\end{array}$ \\
\hline $\begin{array}{l}\text { To differentiate fiber tip } \\
\text { pens }\end{array}$ & $\begin{array}{l}48 \text { fiber-tip pens } \\
\text { (black, red, green } \\
\text { and blue) }\end{array}$ & ATR-FTIR 3 & $\begin{array}{c}\mathrm{PCA}^{20} \text { and } \\
\mathrm{LDA}^{26}\end{array}$ & $\begin{array}{c}\text { Unscrambler X } \\
10.5 .1\end{array}$ & Yadav et al. [37] \\
\hline $\begin{array}{l}\text { To differentiate black } \\
\text { pens }\end{array}$ & $\begin{array}{l}55 \text { different classes } \\
\text { of black pens }\end{array}$ & $\begin{array}{l}\text { VSC }^{\circledR} 6000^{16} \text { and } \\
\text { LC/MS-TOF }^{17}\end{array}$ & PLS-DA ${ }^{28}$ & MATLAB $^{\circledR}$ & Silva et al. [38] \\
\hline $\begin{array}{l}\text { To differentiate blue } \\
\text { pens }\end{array}$ & $\begin{array}{l}25 \text { different classes } \\
\text { of blue pens }\end{array}$ & VSC $^{\circledR} 6000^{16}$ & PLS-DA ${ }^{28}$ & MATLAB $^{\circledR}$ & Silva et al. [39] \\
\hline
\end{tabular}

To differentiate pen

To diffe
inks

To differentiate pen

inks

To determine ballpoint

pens crossing lines order

To determine ballpoint pens crossing lines order

To determine gel pens crossing lines order

To determine ballpoint and gel pens crossing lines order
42 blue pen inks from different types and brands

\begin{tabular}{|c|c|}
\hline MIA $^{8} /$ iPhone $^{\circledR}$ & PLS-DA ${ }^{28}$ \\
\hline
\end{tabular}

16 black pen inks from different types and brands

HSI-NIR ${ }^{18}$

$\mathrm{PCA}^{20}$ and $\mathrm{PP}^{33} \quad \mathrm{MATLAB}^{\circledR}$

Pereira et al. [41]

3 black ballpoint pens

ToF-SIMS ${ }^{19}$

$\mathrm{PCA}^{20}$ and

MATLAB ${ }^{\circledR}$

$\mathrm{MCR}^{34}$

\begin{tabular}{|c|c|c|c|c|}
\hline $\begin{array}{l}7 \text { blue ballpoints pen } \\
\text { brands }\end{array}$ & VSC6000 ${ }^{\circledR 16}$ & MCR-ALS ${ }^{35}$ & MATLAB $^{\circledR}$ & $\begin{array}{c}\text { Martins et al. } \\
{[43]}\end{array}$ \\
\hline $\begin{array}{l}8 \text { blue and black gel } \\
\text { pens from different } \\
\text { brands }\end{array}$ & $\begin{array}{c}\text { Raman } \\
\text { Spectroscopy }\end{array}$ & $\begin{array}{l}\text { MCR-ALS }{ }^{35} \text { and } \\
\text { PLS-DA }\end{array}$ & MATLAB $^{\circledR}$ & Brito et al. [44] \\
\hline $\begin{array}{l}21 \text { black ballpoint } \\
\text { and gel pens from } \\
\text { different brands }\end{array}$ & HSI-NIR ${ }^{18}$ & $\begin{array}{c}\mathrm{PCA}^{20}, \mathrm{MCR}- \\
\mathrm{ALS}^{35} \text { and PLS- } \\
\mathrm{DA}^{28}\end{array}$ & MATLAB $^{\circledR}$ & Brito et al. [45] \\
\hline
\end{tabular}

N/I: not informed; 'Vis-MSP: Visivel-Microspectrophotometry; ${ }^{2}$ DI-MS: Direct-Injection Mass Spectrometry; ${ }^{3}$ ATR-FTIR: Attenuated Total Reflectance Fourier-Transform Infrared Spectroscopy; ${ }^{4}$ UV-Vis-NIR: Ultraviolet-Visible-Near-Infrared Spectroscopy; ${ }^{5}$ UV-Vis: Ultraviolet-Visible Spectroscopy; ${ }^{6}$ IR: Infrared; ' $\mathrm{HPTLC}$ : High-Performance Thin Layer Chromatography; ${ }^{8}$ MIA: Multivariate Image Analysis; ${ }^{9}$ Orbitrap MS: Orbitrap Mass Spectrometry; ${ }^{10}$ FTIR: Reflectance Fourier-Transform Infrared Spectroscopy; ${ }^{11}$ PS-MS: Paper Spray Mass Spectrometry; ${ }^{12}$ LA-ICP-MS: Laser Ablation Inductively Coupled Plasma Mass Spectrometry; ${ }^{13}$ UV-NIR: Ultraviolet-Near-Infrared Spectroscopy; ${ }^{14} \mathrm{HSI}$ : Hyperspectral imaging; ${ }^{15} \mathrm{LIBS}$ : Laser-Induced Breakdown Spectroscopy; ${ }^{16}$ VSC ${ }^{\circledR 6000}$ : Video Spectral Comparator; ${ }^{17}$ LC/MS-TOF: Liquid Chromatography Quadrupole Time-of-Flight; ${ }^{18} \mathrm{HSI}-\mathrm{NIR}$ : Hyperspectral Imaging Near-Infrared; ${ }^{19} \mathrm{ToF}-\mathrm{SIMS}$ : Time-of-Flight Secondary lon Mass Spectrometry; ${ }^{20} \mathrm{PCA}$ : Principal Component Analysis; ${ }^{21} \mathrm{HCA}$ : Hierarchical Cluster Analysis; ${ }^{22}$ OPLS: Orthogonal Partial Least Squares; ${ }^{23}$ ULT: Unsupervised Linkage Threshold; ${ }^{24}$ PLS: Partial Least Squares; ${ }^{25} \mathrm{MLR}$ : Multiple-linear Regression; ${ }^{26}$ LDA: linear discriminate analysis; ${ }^{27}$ PLSR: Partial Least Squares Regression; ${ }^{28}$ PLS-DA: Partial Least Squares-Discriminant Analysis; ${ }^{29}$ MF-ICA: Mean-field Approach Independent Component Analysis; ${ }^{30}$ MANOVA: Multivariate Analysis of Variance; ${ }^{31} \mathrm{DA}$ : Discriminant Analysis; ${ }^{32}$ SAM: Spectral Angle Mapper; ${ }^{33} \mathrm{MCR}$ : Multivariate Curve Resolution; ${ }^{33} \mathrm{PP}$ : Projection Pursuit; ${ }^{34} \mathrm{MCR}$-ALS: Multivariate Curve Resolution with Alternating Least Squares. 
Gorziza, R. P.; González, M.; de Carvalho, C. M. B.; Ortiz, R. S.; Ferrão, M. F.; Limberger, R. P.

Table II. Studies concerning printed documents analysis and multivariate analysis/chemometrics approaches

\begin{tabular}{|c|c|c|c|c|c|}
\hline Main Objectives & Samples & $\begin{array}{l}\text { Analytical } \\
\text { Method }\end{array}$ & $\begin{array}{c}\text { Multivariate } \\
\text { Analysis }\end{array}$ & Software & Reference \\
\hline $\begin{array}{l}\text { To characterize lard on } \\
\text { food packages inks }\end{array}$ & Not specified & $\mathrm{FTIR}^{1}$ & $\begin{array}{l}\mathrm{PCA}^{15} \text { and } \\
\mathrm{SIMCA}^{16}\end{array}$ & $\begin{array}{c}\text { Unscrambler X } \\
10.3\end{array}$ & $\begin{array}{l}\text { Ramli et al. } \\
\text { [46] }\end{array}$ \\
\hline $\begin{array}{l}\text { To identify counterfeit } \\
\text { pharmaceutical } \\
\text { packages }\end{array}$ & $\begin{array}{l}124 \text { paperboard } \\
\text { packages } \\
\text { representing the } \\
\text { secondary packaging } \\
\text { of } 6 \text { pharmaceutical } \\
\text { products }\end{array}$ & $\begin{array}{c}\text { LIBS }^{2} \text { and ATR- } \\
\text { FTIR }^{3}\end{array}$ & $\begin{array}{l}\mathrm{PCA}^{15}, \mathrm{KNN}^{17} \\
\text { and } \mathrm{LDA}^{18}\end{array}$ & JMP Pro 14 & $\begin{array}{l}\text { Haase et al. } \\
\text { [47] }\end{array}$ \\
\hline $\begin{array}{l}\text { To discriminate } \\
\text { authentic and } \\
\text { counterfeit stamps }\end{array}$ & $\begin{array}{l}8 \text { counterfeits } \\
\text { revenue stamps }\end{array}$ & $\begin{array}{c}\mathrm{XRF}^{4} \\
\text { Spectroscopy }\end{array}$ & $\mathrm{PCA}^{15}$ & Pirouette 3.11 & $\begin{array}{l}\text { Perez et al. } \\
\text { [48] }\end{array}$ \\
\hline $\begin{array}{l}\text { To classify pigments } \\
\text { and inks }\end{array}$ & $\begin{array}{l}10 \text { blue and black } \\
\text { inks on paper }\end{array}$ & $\begin{array}{l}\text { Raman } \\
\text { Spectroscopy } \\
\text { and } \text { LIBS }^{2}\end{array}$ & $\begin{array}{c}\mathrm{PCA}^{15}, \\
\mathrm{SIMCA}^{16}, \\
\text { PLS-DA }^{19} \text {, and } \\
\text { SVM }^{20}\end{array}$ & $\begin{array}{l}\text { Unscrambler } X \\
9.8 \text { and } 10.1\end{array}$ & $\begin{array}{l}\text { Hoehse et al. } \\
\text { [49] }\end{array}$ \\
\hline $\begin{array}{l}\text { To create a database } \\
\text { of } 76 \text { toners, } 78 \text { inkjets } \\
\text { inks, } 79 \text { offset inks, and } \\
86 \text { intaglio inks }\end{array}$ & $\begin{array}{l}319 \text { specimens } \\
\text { representing four } \\
\text { major types of } \\
\text { printing inks }\end{array}$ & $\begin{array}{c}\text { FTIR }^{1}, \text { SEM- } \\
\text { EDS }^{5} \text {,LA-ICP- } \\
\text { MS }^{6}, \text { DART-MS } \\
\text { and } \text { Py-GC-MS }\end{array}$ & PLS-DA $^{19}$ & $\begin{array}{l}\text { SYSTAT, JMP, } \\
\text { Excel 2011, Plot } \\
\text { for mac OSX, } \\
\text { Mathematica and } \\
\text { MATLAB }^{\circledR}\end{array}$ & $\begin{array}{c}\text { Trejos et al. } \\
\text { [50] }\end{array}$ \\
\hline $\begin{array}{l}\text { To discriminate among } \\
\text { printing devices from } \\
\text { laser, inkjet, and } \\
\text { photocopier machines }\end{array}$ & 45 printout samples & ATR-FTIR ${ }^{3}$ & $\begin{array}{l}\mathrm{PCA}^{15}, \mathrm{HCA}^{21} \\
\text { and } \mathrm{LDA}^{18}\end{array}$ & SPSS-20 ${ }^{\circledR}$ & $\begin{array}{c}\text { Kumar et al. } \\
{[51]}\end{array}$ \\
\hline $\begin{array}{l}\text { Classification of inkjet } \\
\text { prints }\end{array}$ & 22 different printers & FT-NIR ${ }^{9}$ & $\begin{array}{l}\mathrm{DA}^{22}, \mathrm{LDA}^{18} \\
\text { and } \mathrm{QDA}^{23}\end{array}$ & $\begin{array}{c}\text { Unscrambler X } \\
10.3\end{array}$ & $\begin{array}{c}\text { Oravec et al. } \\
\text { [52] }\end{array}$ \\
\hline $\begin{array}{l}\text { To correlate toners of } \\
\text { unknown origin }\end{array}$ & 10 black toners & $\mathrm{NIR}^{10}$ & $\mathrm{PCA}^{15}$ & $\begin{array}{l}\text { V-PARVUS } 2009 \\
\text { package }\end{array}$ & $\begin{array}{c}\text { Materazzi et al. } \\
{[53]}\end{array}$ \\
\hline $\begin{array}{l}\text { To discriminate and } \\
\text { classify toners }\end{array}$ & $\begin{array}{l}40 \text { different black } \\
\text { toners sources each } \\
\text { for laser printer } \\
\text { and photocopier } \\
\text { machines }\end{array}$ & FE-SEM-EDS ${ }^{11}$ & $\begin{array}{l}\mathrm{PCA}^{15}, \mathrm{HCA}^{21} \\
\text { and } \mathrm{LDA}^{18}\end{array}$ & $\begin{array}{l}\text { Microsoft Excel } \\
\text { and SPSS-20 }\end{array}$ & $\begin{array}{l}\text { Verma et al. } \\
\qquad 54]\end{array}$ \\
\hline $\begin{array}{l}\text { To discriminate and } \\
\text { classify toners }\end{array}$ & $\begin{array}{l}100 \text { samples from } \\
\text { printouts taken } \\
\text { from laser printers } \\
\text { and photocopier } \\
\text { machines }\end{array}$ & UV-Vis ${ }^{12}$ & $\begin{array}{l}\mathrm{PCA}^{15}, \mathrm{DP}^{24} \\
\text { and Varimax } \\
\text { Rotation }\end{array}$ & SPSS-20 ${ }^{\circledR}$ & $\begin{array}{l}\text { Verma et al. } \\
\qquad[55]\end{array}$ \\
\hline $\begin{array}{l}\text { To differentiate black } \\
\text { toners }\end{array}$ & $\begin{array}{l}49 \text { types of laser } \\
\text { printers of latter } \\
\text { brands }\end{array}$ & $\mathrm{FTIR}^{1}$ & $\begin{array}{l}\mathrm{PCA}^{15} \text { and } \\
\text { MANOVA }^{25}\end{array}$ & Unscrambler X & $\begin{array}{l}\text { Gál et al. } \\
\text { [56] }\end{array}$ \\
\hline $\begin{array}{l}\text { To discriminate paper } \\
\text { brands and crossing } \\
\text { lines order }\end{array}$ & $\begin{array}{l}12 \text { different paper } \\
\text { brands, } 2 \text { toners and } \\
3 \text { blue ballpoint pens }\end{array}$ & $\mathrm{FTIR}^{1}$ and $\mathrm{AFM}^{13}$ & $\mathrm{PCA}^{15}$ & $\begin{array}{c}\text { Unscrambler X } \\
10.3\end{array}$ & $\begin{array}{l}\text { Farid et al. } \\
\qquad[57]\end{array}$ \\
\hline $\begin{array}{l}\text { To distinguish } \\
\text { documents by papers } \\
\text { and colorants }\end{array}$ & $\begin{array}{l}8 \text { printer papers, } \\
\text { marker pen inks, } \\
\text { inkjets and printer } \\
\text { toners }\end{array}$ & $\mathrm{NIR}^{10}$ & $\mathrm{PCA}^{15}$ & MATLAB ${ }^{\circledR}$ & $\begin{array}{c}\text { Sugawara et al. } \\
{[58]}\end{array}$ \\
\hline
\end{tabular}


Braz. J. Anal. Chem., 2022, 9 (34), pp 35-51.

Table II. Studies concerning printed documents analysis and multivariate analysis/chemometrics approaches (Cont)

\begin{tabular}{llcccc}
\hline Main Objectives & Samples & $\begin{array}{c}\text { Analytical } \\
\text { Method }\end{array}$ & $\begin{array}{c}\text { Multivariate } \\
\text { Analysis }\end{array}$ & Software & Reference \\
\hline $\begin{array}{l}\text { To determine the } \\
\text { chronological order of } \\
\text { crossing lines }\end{array}$ & $\begin{array}{l}\text { 1 inkjet, 1 toner, 20 } \\
\text { blue ballpoint pens, } \\
16 \text { rollerball pens, 16 } \\
\text { felt-tip pens and } 8 \mathrm{gel} \\
\text { pen inks }\end{array}$ & MIA $^{14} /$ PPhone $^{\circledR}$ & PLS-DA $^{19}$ & MATLAB $^{\circledR}$ & $\begin{array}{c}\text { Valderrama et al. } \\
{[59]}\end{array}$ \\
\hline
\end{tabular}

1FTIR: Fourier-Transform Infrared Spectroscopy; ${ }^{2}$ LIBS: Laser-Induced Breakdown Spectroscopy; ${ }^{3}$ ATR-FTIR: Attenuated Total Reflectance Fourier-Transform Infrared Spectroscopy; ${ }^{4} \mathrm{XRF}$ : X-Ray Fluorescence; ${ }^{5}$ SEM-EDS: Scanning Electron Microscopy-Energy Dispersive X-Ray Spectroscopy; ${ }^{6}$ LA-ICP-MS: Laser Ablation Inductively Coupled Plasma Mass Spectrometry; ${ }^{7}$ DART-MS: Direct Analysis in Real Time Mass Spectrometry; ${ }^{8} \mathrm{Py}-\mathrm{GC}-\mathrm{MS}$ : Analytical Pyrolysis combined with Gas Chromatography-Mass Spectrometry; ${ }^{9} \mathrm{FT}$-NIR: Fourier Transform NearInfrared; ${ }^{10} \mathrm{NIR}$ : Near-Infrared; ${ }^{11} \mathrm{FE}-\mathrm{SEM}$-EDS: Field Emission Scanning Electron Microscopy-Energy Dispersive X-Ray Spectroscopy; ${ }^{12} \mathrm{UV}-V i s:$ Ultraviolet-Visible Spectroscopy; ${ }^{13} \mathrm{AFM}$ : Atomic Force Microscopy; ${ }^{14} \mathrm{MIA}$ : Multivariate Image Analysis; ${ }^{15} \mathrm{PCA}$ : Principal Component Analysis; ${ }^{16}$ SIMCA: Soft Independent Modelling by Class analogy; ${ }^{17} \mathrm{KNM}$ : K-nearest neighbors; ${ }^{18} \mathrm{LDA}$ : Linear Discriminant Analysis; ${ }^{19} \mathrm{PLS}-\mathrm{DA}:$ Partial Least Squares Discriminant Analysis; ${ }^{20}$ SVM: Support Vector Machines; ${ }^{21} \mathrm{HCA}$ : Hierarchical Cluster Analysis; ${ }^{22} \mathrm{DA}$ : Discriminant Analysis; ${ }^{23}$ QDA: Non-Linear (Quadratic) Classification Analyses; ${ }^{24}$ DP: Discrimination Power; ${ }^{25}$ MANOVA: Multivariate Analysis of Variance.

Table III. Studies concerning paper analysis and multivariate analysis/chemometrics approaches

\begin{tabular}{|c|c|c|c|c|c|}
\hline Main Objectives & Samples & $\begin{array}{l}\text { Analytical } \\
\text { Method }\end{array}$ & $\begin{array}{c}\text { Multivariate } \\
\text { Analysis }\end{array}$ & $\begin{array}{c}\text { Software for } \\
\text { Analysis }\end{array}$ & Reference \\
\hline $\begin{array}{l}\text { To characterize and } \\
\text { to discriminate paper } \\
\text { relics }\end{array}$ & 15 types of paper & ATR-FTIR ${ }^{1}$ & 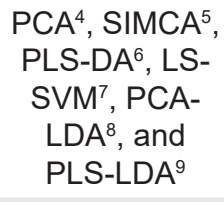 & MATLAB $^{\circledR}$ & $\begin{array}{l}\text { Xia et al. } \\
\text { [60] }\end{array}$ \\
\hline $\begin{array}{l}\text { To characterize and to } \\
\text { discriminate papers. }\end{array}$ & $\begin{array}{l}24 \text { different kinds } \\
\text { of writing/printing } \\
\text { papers }\end{array}$ & ATR-FTIR ${ }^{1}$ & $\mathrm{PCA}^{4}$ & SPSS-16 ${ }^{\circledR}$ & $\begin{array}{c}\text { Kumar et al. } \\
\text { [62] }\end{array}$ \\
\hline Paper dating & $\begin{array}{l}\text { Reports from } 15 \\
\text { different years }\end{array}$ & FTIR $^{2}$ & $\begin{array}{l}\mathrm{PLS}^{11} \text { and } \\
\mathrm{PCA}^{4}\end{array}$ & MATLAB $^{\circledR}$ & $\begin{array}{l}\text { Silva et al. } \\
\text { [65] }\end{array}$ \\
\hline Paper dating & $\begin{array}{l}6 \text { samples of } \\
\text { common papers }\end{array}$ & ATR-FTIR ${ }^{1}$ & $\begin{array}{l}\mathrm{CE}^{12}, \mathrm{MLR}^{13} \\
\text { and } \mathrm{PLSR}^{14}\end{array}$ & SPSS-20 ${ }^{\circledR}$ & $\begin{array}{c}\text { Sharma et al. } \\
{[66]}\end{array}$ \\
\hline Paper dating & $\begin{array}{l}\text { Several types of } \\
\text { historic paper }\end{array}$ & $\begin{array}{l}\text { THz time-domain } \\
\text { spectroscopy }\end{array}$ & $\mathrm{PLS}^{11}$ & Unscrambler v.9.7 & $\begin{array}{c}\text { Trafela et al. } \\
\text { [67] }\end{array}$ \\
\hline
\end{tabular}

N/I: not informed; 'ATR-FTIR: Attenuated Total Reflectance Fourier-Transform Infrared Spectroscopy; ${ }^{2}$ FTIR: Fourier-Transform Infrared Spectroscopy; ${ }^{3} \mathrm{Py}-\mathrm{GC}$-MS: Analytical Pyrolysis combined with Gas Chromatography/Mass Spectrometry; ${ }^{4} \mathrm{PCA}$ : Principal Component Analysis; ${ }^{5}$ SIMCA: Soft Independent Modelling by Class Analogy; ${ }^{6}$ PLS-DA: Partial Least Squares with Discriminant Analysis; ${ }^{7}$ LS-SVM: Least squares support vector machines; ${ }^{8}$ PCA-LDA: Principal Component Analysis-Linear Discrimination Analysis; ${ }^{9}$ PLS-LDA: Partial Least Squares-Linear Discrimination Analysis; ${ }^{10}$ SPLS: Sparse Partial Least Squares; ${ }^{11} \mathrm{PLS}$ : Partial Least Squares; ${ }^{12} \mathrm{CE}$ : Curve Estimation; ${ }^{13} \mathrm{MLR}$ : Multiple Linear Regression; ${ }^{14}$ PLSR: Partial Least Squares Regression. 
Table IV. Studies concerning banknotes analysis and multivariate analysis/chemometrics approaches

\begin{tabular}{|c|c|c|c|c|c|}
\hline Main Objectives & Samples & $\begin{array}{l}\text { Analytical } \\
\text { Method }\end{array}$ & $\begin{array}{c}\text { Multivariate } \\
\text { Analysis }\end{array}$ & Software & Reference \\
\hline $\begin{array}{l}\text { Classification of } \\
\text { banknotes }\end{array}$ & $\begin{array}{l}4 \text { authentic and } 12 \\
\text { falsified Brazilian } \\
\text { banknotes }\end{array}$ & $\begin{array}{c}\mathrm{MIA}^{1} / \\
\text { Smartphone }\end{array}$ & $\mathrm{PCA}^{2}$ & Photometrix $\mathrm{PRO}^{\circledR}$ & $\begin{array}{c}\text { Vittorazzi et al. } \\
\text { [68] }\end{array}$ \\
\hline $\begin{array}{l}\text { Characterization of } \\
\text { banknotes }\end{array}$ & $\begin{array}{l}42 \text { counterfeit } \\
\text { Brazilian banknotes }\end{array}$ & $\begin{array}{l}\text { Portable X-ray } \\
\text { fluorescence } \\
\text { and Raman } \\
\text { Spectroscopy }\end{array}$ & $\begin{array}{l}\mathrm{PCA}^{2} \text { and } \\
\mathrm{PLS}^{3}\end{array}$ & MATLAB $^{\circledR}$ & $\begin{array}{c}\text { Rodrigues et al. } \\
\text { [69] }\end{array}$ \\
\hline $\begin{array}{l}\text { Characterization of } \\
\text { banknotes }\end{array}$ & $\begin{array}{l}1 \text { Dollar bill, } 1 \text { Euro } \\
\text { bill and } 6 \text { Real bills }\end{array}$ & $\begin{array}{l}\text { Portable X-ray } \\
\text { Fluorescence }\end{array}$ & $\mathrm{PCA}^{2}$ & Not informed & $\begin{array}{c}\text { Appoloni et al. } \\
{[70]}\end{array}$ \\
\hline $\begin{array}{l}\text { To differentiate } \\
\text { authentic and } \\
\text { counterfeit banknotes }\end{array}$ & $\begin{array}{l}\text { Original and } \\
\text { counterfeit Brazilian } \\
\text { banknotes }\end{array}$ & $\begin{array}{l}\text { Raman } \\
\text { Spectroscopy }\end{array}$ & PLS-DA ${ }^{4}$ & MATLAB $^{\circledR}$ & $\begin{array}{c}\text { Almeida et al. } \\
\text { [71] }\end{array}$ \\
\hline
\end{tabular}

1MIA: Multivariate Image Analysis; ${ }^{2}$ PCA: Principal Component Analysis; ${ }^{3}$ PLS: Partial Least Squares; ${ }^{4}$ PLS-DA: Partial Least Squares with Discriminant Analysis.

Overall, spectroscopic techniques are the most studies methods to acquire chemical data from questioned documents, prior to multivariate analysis (Figure 3). Mass spectrometry, chromatography, thermo gravimetric and $\mathrm{x}$-ray-based techniques were also studied. Besides a few exceptions, most of these methods are non-destructive techniques, which is an advantage in the questioned documents area, maintaining documents integrity for counterproof. In addition, the method variability allows for many possibilities of documents analysis across different Laboratories. However, it is necessary for Forensic Experts to understand the chemometrics data analysis to conduct appropriate results interpretation and data presentation in reports.

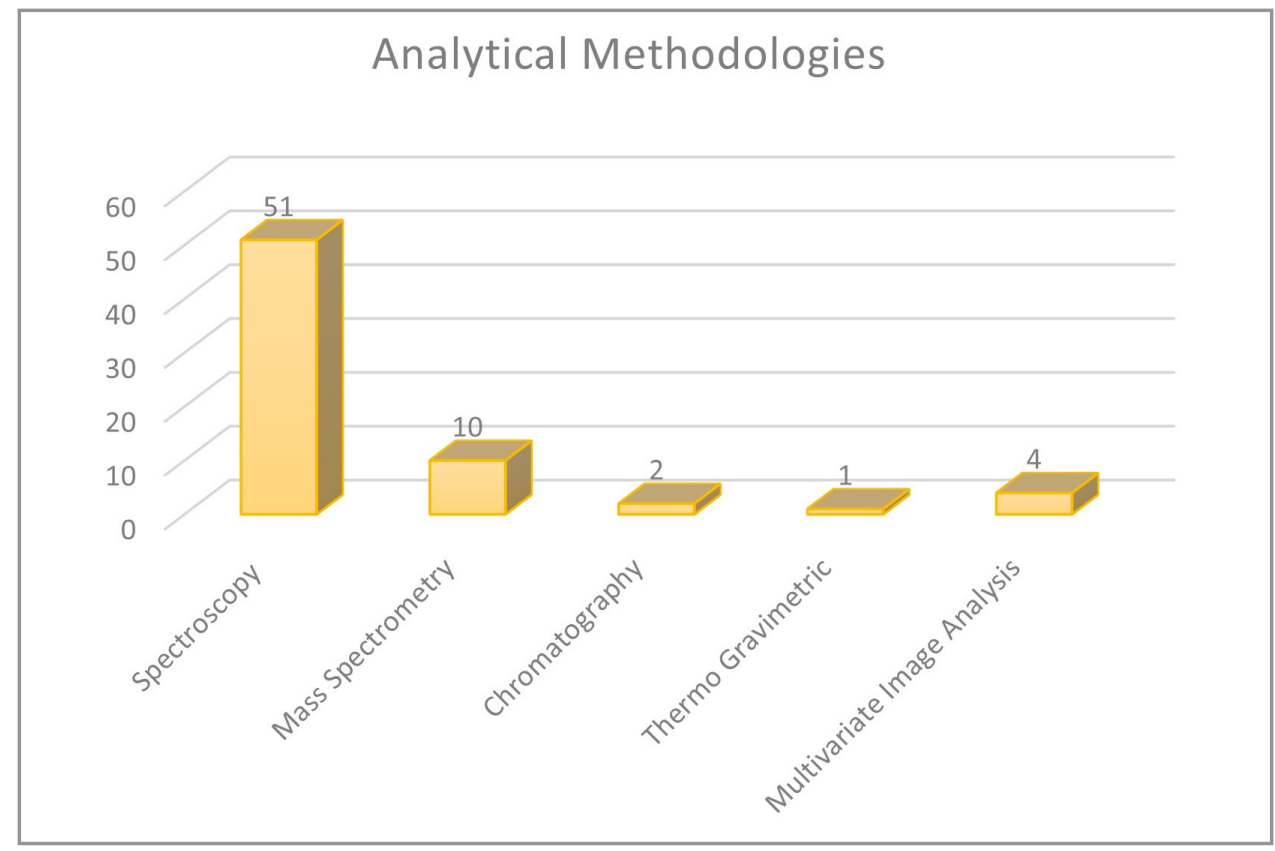

Figure 3. Analytical methodologies applied on questioned documents to capture chemical data. 
Prior to multivariate analysis, all the chemical data produced with analytical methodologies (or multivariate image analysis) need to be organized into a matrix. For instance, this matrix associates each sample in lines, while the variables are displayed in columns [72,73]. Thus, sample pre-processing can be performed to minimize undesirable variables, which could rise during data acquisition and interfere with the analysis results [72,73]. Figure 4 shows the most applied multivariate analysis techniques following analytic methodologies.

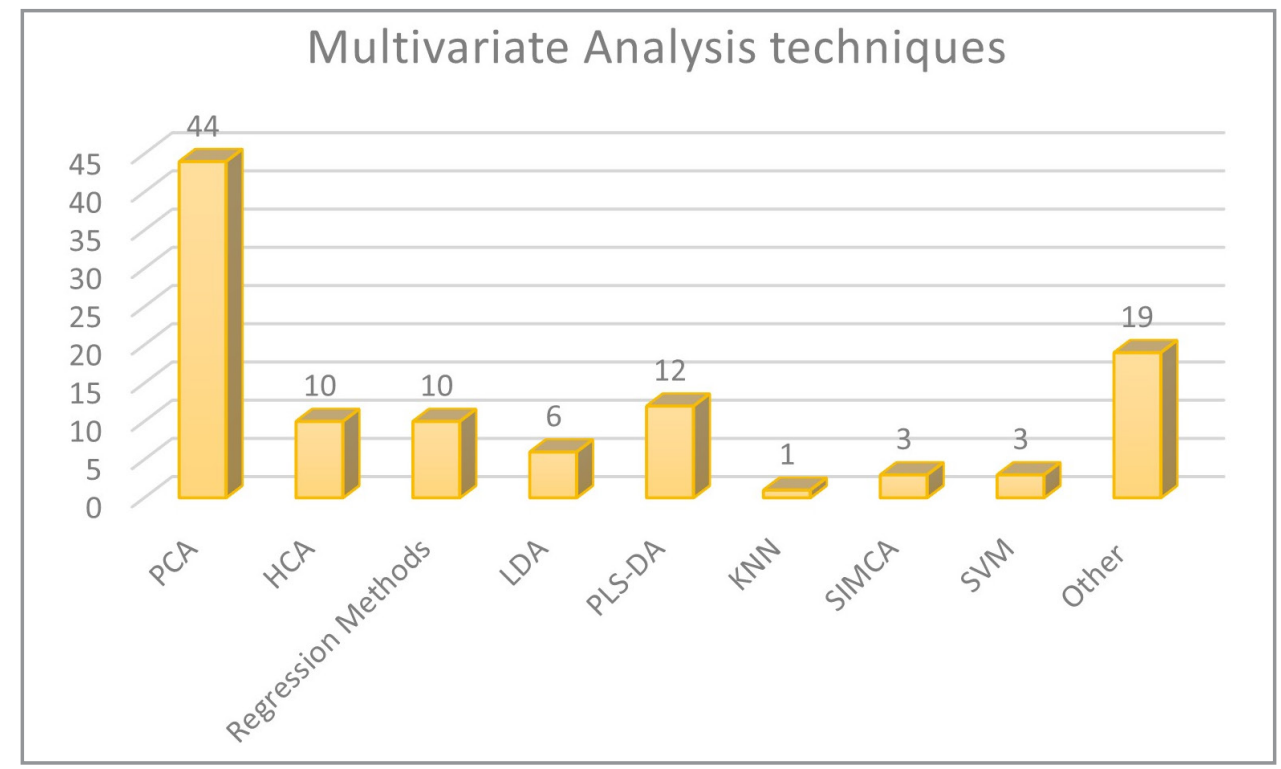

Figure 4. Multivariate analysis techniques involved on data examination from questioned documents.

Multivariate analysis/chemometric techniques can be divided at: a) unsupervised pattern recognition techniques, including principal component analysis (PCA) and hierarchical cluster analysis (HCA); b) regression methods, including partial least squares (PLS) and multiple linear regression (MLR); c) supervised pattern recognition techniques, including linear discriminant analysis (LDA), partial least squares discriminant analysis (PLS-DA), k-nearest neighbor (kNN), soft independent modeling of class analogy (SIMCA) and support vector machine (SVM). Other techniques included mean-field approach independent component analysis (MF-ICA), multivariate analysis of variance (MANOVA), spectral angle mapper (SAM), multivariate curve resolution (MCR) and multivariate curve resolution with alternating least squares (MCR-ALS), among others. A summary of these techniques is shown in Table V. 
Table V. Concepts of the main chemometric techniques applied in questioned document analysis

\begin{tabular}{lc}
\hline $\begin{array}{l}\text { Method } \\
\text { Classification }\end{array}$ & $\begin{array}{c}\text { Multivariate } \\
\text { Analysis }\end{array}$ \\
\hline
\end{tabular}

the relation between samples. It is a type of controlled loss of information that is compensated by a better understanding of the data

PCA inside the data set. This method allows for visualizing and interpreting the differences between the variables and examining the relations

UNSUPERVISED

PATTERN

RECOGNITION

TECHNIQUES that may exist between the samples. The method is also capable to detect samples with an anomalous behavior: the data projection makes the differences evident [72].

This method is originated from numerical taxonomy. It is useful for reducing the dimensionality of large data sets by gathering samples into groups of samples that are most similar to each other. Thus, both internal homogeneity within groups and heterogeneity between groups are maximized. The results are presented in a hierarchical tree denominated dendrogram, and the tree branches show a degree of similarity between the samples. This similarity is calculated over a distance: similar samples from one group have a shorter distance between them when compared to samples from other groups [73].

It is a biased method that applies factor analysis, and it is the most popular regression method in chemometrics. The purpose of this technique is to estimate the space of original measures into one of reduced size. The model is built in a single step, in which the technique is to estimate the space of original measures into one of reduced size. The model is built in a single step, in which the
information from matrix " $X$ " and values of interest are considered during the data decomposition and compression. Some restriction is imposed on the decomposition of matrix " $X$ " (of samples and variables), directing it to a solution whose target is the property of interest. This is justifiable, if the values of the property of interest are reliable [73].

REGRESSION

METHODS

The general purpose of this method is to quantitate the relationship between several independent or predictor variables and a dependent

$\begin{array}{ll}\text { MLR } & \text { The general purpose of this method is to quantitate the relationship between several independent or predictor variables and a } \\ \text { variable. The MLR model is built with descriptive variables using the least squares methods to minimize the residuals [74]. }\end{array}$

it is a linear combination of the sample's set original attributes, characterized by producing the maximum separation between two populations. The main objectives of this method are a) to confirm whether the groups are correctly discriminated, b) to classify unknown

LDA observations, and c) to verify which are the most important variables for the discrimination of these groups. It takes a different approach
to consider the existence of classes for the data; it involves projecting the data distribution probability on the graphic's axes. Therefore,

LDA observations, and c) to verify which are the most important variables for the discrimination of these groups. It takes a different approach
to consider the existence of classes for the data; it involves projecting the data distribution probability on the graphic's axes. Therefore, the method not only maintains but it also highlights a linear separation of the data, if it exists [75].

It is a method that determines which class an unknown sample belongs, based on the information provided to the system. The method applies multivariate regression by partial least squares (PLS), which has been previously discussed. PLS is an inverse calibration

SUPERVISED

PATTERN

RECOGNITION

PLS-DA method, in which a direct relationship is sought between the instrumental response (matrix $X)$ and the property of interest (matrix $Y$ or vector $\mathrm{y}$ ). The classification model is built using the same PLS model. However, in PLS-DA the property of interest is a categorical variable that describes the sample's class assignment. Generally, a value of 1 is assigned to the class of interest and a value of 0 is assigned to the other class [76].

This deterministic model is defined based on a training set, followed by the classification model construction. When building the model, each sample of the training set is deleted and then classified based on the remaining samples. The distances between the excluded sample and the remaining samples of the training set are calculated in the dimensional space. The excluded sample is then classified according to a majority of "votes" from its closest neighbors, and the sample is attributed to the most voted class. This process is applied to all training samples, with a summary of successful analyzes and errors at the end [77]. 
Table V. Concepts of the main chemometric techniques applied in questioned document analysis (Continuation)

\section{Method Multivariate \\ Classification Analysis}

This method assumes that the measured values for a group of similar samples will tend towards a uniform and modellable distribution. By increasing the number of samples, the uniform distribution becomes more visible. The probabilistic distribution allows for estimating the degree of certainty in the classification. This model allocates the main component model to be adjusted to each class in the training set, giving rise to a classifier for each one. The number of factors, suitable for modeling each class, can be determined by doing a crossvalidation to maximize the predictive capacity of the individual models in relation to the training set. If it is necessary to include a new class, it is possible to build an independent model for each class by adding it to the existing model, without having to repeat the entire modeling process [77].

SUPERVISED

PATTERN

RECOGNITION

TECHNIQUES

SIMCA

It is a supervised machine learning algorithm that can be employed for both classification and regression purposes. It is based on the idea of finding a hyperplane that best divides a dataset into two classes. Intuitively, the further from the hyperplane the data points lie, the more confident the results are, which means they are correctly classified. Therefore, the goal is for the data points as far away from the hyperplane as possible, while still stands on the correct side of it. When adding new testing data, the side of the hyperplane in which it lands will define the data class [78].

Independent component analysis (ICA) is a computational method for separating a multivariate signal into additive subcomponents. This is done by assuming that the subcomponents are non-Gaussian signals and that they are statistically independent from each other. In MF-ICA we derive an expectation-maximization algorithm, in which the expectation step is performed using different medium field (MF) approaches: variational, linear response, and adaptive TAP (Thouless, Anderson and Palmer). The MF theories produce estimates of later source correlations of increasing quality, needed for the maximization step in the estimate for the multivariate signal and the noise [79].

MF-ICA

It is a test to perform the relationship analysis between several response variables and a common set of predictors at the same time. It requires continuous response variables and categorical predictors. MANOVA has several important advantages over performing several ANOVAs, one response variable at a time [80].

OTHER

METHODS

It is an algorithm that allows for the measurement of spectral similarity between two spectra. This is expressed as a numerical scale (from 0-no similarity to 1-identical spectra). In this approach, selected spectra are treated as vectors in n-dimensional space, in which the number of dimensions is equal to the number of recorded spectral lines. This allows for the calculation of the spectral angle. It is worth mentioning that the SAM method is resistant to illumination variation [36].

The MCR method was first created for process analysis purposes, but nowadays it is also applied to non-evolutionary multicomponent systems. Thus, the MCR method was created and classified as a two-way data analysis method, i.e., a valid method to analyze single

data matrices. However, the general applications of MCR relates to the possibility to work with multi-way and multiset data structures, i.e., with several data tables simultaneously [81]. It is an algorithm that solves the MCR basic bilinear model, using a constrained Alternating Least Squares algorithm. The constraints

MCR-ALS improve the profiles interpretability in pure spectra matrices and the related concentration profiles for each of the compounds in the system [81]. 
As we can observe in Tables I, II, III and IV, different software can applied to perform multivariate analysis or chemometrics, and MATLAB ${ }^{\circledR}$, SPSS$^{2} 0^{\circledR}$, Unscrambler $^{\circledR}$, SIMCA $^{\circledR}$, Minitab ${ }^{\circledR}$, Microsoft Excel $^{\circledR}$, Chemostat ${ }^{\circledR}$, and Photometrix $\mathrm{PRO}^{\circledR}$ are the most applied.

In this review, a substantial number of papers applying chemometrics approaches in questioned documents were identified and summarized. These studies show a growing trend for the chemometrics importance to the field, over the past ten years. In this matter, it is crucial for questioned documents experts to understand how to perform data analysis and how to present the results for forensic purposes. For this reason, this review included a brief summary of the most applied chemometric techniques, that should be known by forensic experts. Deviterne-Lapeyre [3] have discussed the challenge of chemical data evaluation for a document examiner scientist. The author emphasized that experts should understand principles and theories about chemometrics, to better explain the results.

Most of the studies herein presented used chemometrics approaches for classification and/or differentiation of samples. Considering the comparison examination, while analytical methods can provide accurate inks and paper chemical data, chemometrics analysis can increase the discriminating power of these techniques [3]. However, research articles should also include intra-variability analysis as a goal, in order to demonstrate the methods limitations as well [3]. Another important role of chemometrics in questioned documents area is the development of databases [3], especially for samples identification, such as pen or paper brands. However, only one of the 60 articles in this review have aimed for a database creation, for printing inks [50]. This is a promising topic for research in questioned documents applying chemometrics. A few articles applied chemometrics for ageing studies of pen inks [12-18] and paper [63-67], but chemical document dating remains as a research topic until a complete standardization is performed [3].

Overall, ballpoint pen inks are the most studied topic of chemometric approaches in questioned documents. Although different types of pen inks, printing inks, paper and banknotes were also studied, these topics are not completely explored and further research using different analytical methodologies and chemometrics data analysis can be performed.

\section{CONCLUSION}

This review compiled a significant number of papers that applied chemometrics in the questioned documents area, describing a brief summary of the most applied chemometrics techniques. These studies show different analytical methodologies applied in pen inks, printed documents, paper, and banknotes analysis. Regression methods, unsupervised and supervised pattern recognition techniques were applied for data analysis with different purposes in forensic science, such as discrimination and classification of samples, ageing estimation, determination of crossing lines chronological order and counterfeit banknotes identification. Under light of these studies, the importance of chemometrics in questioned documents is highlighted, and this knowledge should be included in forensic experts training. The chemometric approach for databases development and implementation is a promising research topic for questioned documents, as well as inks and paper ageing studies and new analytical methods for non-ballpoint pen inks, printing inks, paper, banknotes and different security documents.

\section{Conflicts of interest}

The authors declare that they have no conflicts of interest.

\section{Acknowledgements}

This work was supported by "Coordenação de Aperfeiçoamento de Pessoal de Nível Superior" (CAPES) and "Conselho Nacional de Desenvolvimento Científico e Tecnológico" (CNPq) Brazilian Institutions. This study is a part of CAPES PRO-FORENSES PROJECT (Processo 23038.006845/2014-91) and part of MCTI/CNPQ/CAPES/FAPS n 16/2014 - Programa INCT, CNPq 465450/2014-8 Brazilian Public Call Project, entitled "Instituto Nacional de Ciência e Tecnologia Forense". The authors wish to thank these institutions. 


\section{REFERENCES}

1. Calcerrada, M.; Garcia-Ruiz, C. Anal. Chim. Acta., 2019, 853, pp 143-166 (https://doi.org/10.1016/j. aca.2014.10.057).

2. LaPorte, G. M. Forensic Chem., 2015, pp 318-353 (https://doi.org/10.1002/9781118897768.ch8).

3. Deviterne-Lapeyre, C. M. Forensic Sci. Int. Synerg., 2020, 2, pp $429-441$ (https://doi.org/10.1016/j. fsisyn.2020.01.012).

4. Trejos, T.; Almirall, J. R. Forensic Sci. Handb., 2020, pp 425-463 (https://doi.org/10.4324/9781315119939-7).

5. Chen, H.; Meng, H.; Cheng, K. Forensic Sci. J., 2002, 1 (1), pp 1-14.

6. Ezcurra, M.; Góngora, J. M. G.; Maguregui, I.; Alonso, R. Forensic Sci. Int., 2010, 197, pp 1-20 (https:// doi.org/10.1016/j.forsciint.2009.11.013).

7. Brito, L. R.; Martins, A. R.; Braz, A.; Chaves, A. B.; Braga, J. W.; Pimentel, M. F. TrAC - Trends Anal. Chem., 2017, 94, pp 54-69 (https://doi.org/10.1016/j.trac.2017.07.005).

8. Gorziza, R. P.; Carvalho, C. M. B.; González, M.; Leal, L. B.; Korndörfer, T.; Ortiz, R. S.; Trejos, T.; Limberger, R. P. Brazilian Journal of Forensic Sciences / Medical Law and Bioethics, 2019, 8, pp 113-138 (https://doi.org/10.17063/bjfs8(3)y2019113).

9. Kowalski, B. R. J. Chem. Inf. Comput. Sci., 1975, 15, pp 201-203 (https://doi.org/10.1021/ci60004a002).

10. International Union of Pure and Applied Chemistry (IUPAC). Compendium of Chemical Terminology, 2009 (https://doi.org/10.1351/goldbook).

11. Kumar, R.; Sharma, V. TrAC - Trends Anal. Chem., 2018, 105, pp 191-201 (https://doi.org/10.1016/j. trac.2018.05.010).

12. Ortiz-Herrero, L.; de Almeida, A. C. A.; Bartolomé, L.; Alonso, M. L.; Maguregui, M. I.; Alonso, R. M.; de Melo, J. S. S. Chemom. Intell. Lab. Syst., 2020, 207, pp 104187 (https://doi.org/10.1016/j. chemolab.2020.104187).

13. Costa, K. F. F.; Brand, G. D.; Grobério, T. S.; Braga, J. W. B.; Zacca, J. J. Microchem. J., 2019, 147, pp 1123-1132 (https://doi.org/10.1016/j.microc.2019.04.034).

14. Carvalho, C. M. B.; Ortiz, R. S.; dos Reis, M.; Ferrão, M. F.; Limberger, R. P. J. Am. Soc. Quest. Doc. Exam. Inc., 2019, pp 19-35.

15. Ortiz-Herrero, L.; Bartolomé, L.; Durán, I.; Velasco, I.; Alonso, M. L.; Maguregui, M. I.; Ezcurra, M. Microchem. J., 2018, 140, pp 158-166 (https://doi.org/10.1016/j.microc.2018.04.019).

16. Sharma, V.; Kumar, R. Microchem. J., 2017, 134, pp 104-113 (https://doi.org/10.1016/J. MICROC.2017.05.014).

17. Sauzier, G.; McGann, J.; Lewis, S. W.; Van Bronswijk, W. Anal. Methods, 2018, 10, pp 5613-5621 (https://doi.org/10.1039/c8ay01418c).

18. Senior, S.; Hamed, E.; Masoud, M.; Shehata, E. J. Forensic Sci., 2012, 7, pp 1087-1093 (https://doi. org/10.1111/j.1556-4029.2012.02091.x).

19. Gorziza, R. P.; Carvalho, C. M. B.; González, M.; Ortiz, R. S.; Helfer, G. A.; Ferrão, M. F.; Limberger, R. P. Brazilian Journal of Forensic Sciences / Medical Law and Bioethics, 2020, 9, pp 331-355 (https:// doi.org/10.17063/bjfs9(3)y2020331).

20. Teixeira, C. A.; Poppi, R. J. Microchem. J., 2019, 144, pp 411-418 (https://doi.org/10.1016/j. microc.2018.10.002).

21. de Carvalho, C. M. B.; Ortiz, R. S.; do Reis, M.; Zamboni, A.; Limberger, R. P.; Ferrão, M. F.; Gontijo, B. V. Forensic Sci. Addict. Res., 2018, 2, pp 1-8 (https://doi.org/10.31031/fsar.2018.02.000537).

22. Kamil, M.; Asri, M.; Desa, W.; Ismail, D. Malaysian J. Forensic Sci., 2015, 6, pp 48-53.

23. Amador, V. S.; Pereira, H. V.; Sena, M.; Augusti, M.; Piccin, R. E. J. Am. Soc. Mass Spectrom., 2017, 28, pp 1965-1976 (https://doi.org/10.1007/s13361-017-1686-z).

24. Sharma, V.; Kumar, R. Vib. Spectrosc., 2017, 92, pp 96-104 (https://doi.org/10.1016/J. VIBSPEC.2017.05.006).

25. Asri, M. N. M.; Desa, W. N. S. M.; Ismail, D. Aust. J. Forensic Sci., 2017, 49, pp 175-185 (https://doi. org/10.1080/00450618.2016.1153712). 
26. Lee, L. C.; Othman, M. R.; Pua, H.; Ishak, A. A. Malaysian J. Anal. Sci., 2012, 3, pp 5-10.

27. Lee, L. C.; Othman, M. R.; Pua, H. Malaysian J. Anal. Sci., 2012, 16, pp 262-272.

28. Kumar, R.; Sharma, V. Spectrochim. Acta - Part A Mol. Biomol. Spectrosc., 2017, 175, pp 67-75 (https://doi.org/10.1016/j.saa.2016.12.008).

29. Halim, M. I. A.; Aziz, N. S. A.; Saim, N.; Osman, R.; Jasmani, H. Malaysian J. Fundam. Appl. Sci., 2014, 8, pp 159-165 (https://doi.org/10.11113/mjfas.v8n3.141).

30. Borba, F. S. L.; Honorato, R. S.; de Juan, A. Forensic Sci. Int., 2015, 49, pp 73-82 (https://doi. org/10.1016/j.forsciint.2015.01.027).

31. Alamilla, F.; Calcerrada, M.; García-Ruiz, C.; Torre, M. Forensic Sci. Int., 2013, 228, pp 1-7 (https://doi. org/10.1016/j.forsciint.2013.01.034).

32. Lee, L. C. Forensic Sci. J., 2014, 13 (1), pp 15-22. Available at: fsjournal.cpu.edu.tw

33. Sharma, V.; Kumar, R.; Devgan, K.; Mishra, P. K.; Ekielski, A.; Kumar, V.; Kumar, V. Spectrosc. Lett., 2018, 51, pp 205-215 (https://doi.org/10.1080/00387010.2018.1452265).

34. Asri, M. N. M.; Noor, N. A. M.; Desa, W. N. S. M.; Ismail, D. Forensic Sci. Forensic Med., 2019, 1, pp 1174-1184 (https://doi.org/10.26735/16586794.2019.004).

35. Al Balah, O. F.; Nassef, O. A. T. Arab J. Nucl. Sci. Appl., 2019, 52 (2), pp 72-78 (https://doi.org/10.21608/ ajnsa.2019.4231.1097).

36. Chlebda, D. K.; Majda, A.; Łojewski, T.; Łojewska, J. Appl. Phys. A Mater. Sci. Process., 2016, 122, pp 1-13 (https://doi.org/10.1007/s00339-016-0494-9).

37. Yadav, P. K.; Sharma, R. M. Vib. Spectrosc., 2020, 108, 103054 (https://doi.org/10.1016/j. vibspec.2020.103054).

38. da Silva, V. A. G.; Talhavini, M.; Zacca, J. J.; Trindade, B. R.; Braga, J. W. B. J. Braz. Chem. Soc., 2014, 25, pp 1552-1564 (https://doi.org/10.5935/0103-5053.20140140).

39. da Silva, V. O. G.; Talhavini, M.; Peixoto, I. C. F.; Zacca, J. J.; Maldaner, A. O.; Braga, J. W. B. Microchem. J., 2014, 116, pp 235-243 (https://doi.org/10.1016/j.microc.2014.05.013).

40. Valderrama, L.; Valderrama, P. Chemom. Intell. Lab. Syst., 2016, 156, pp 188-195 (https://doi. org/10.1016/j.chemolab.2016.06.009).

41. Pereira, J. F. Q.; Silva, C. S.; Braz, A.; Pimentel, M. F.; Honorato, R. S.; Pasquini, C.; Wentzell, P. D. Microchem. J., 2017, 130, pp 412-419 (https://doi.org/10.1016/j.microc.2016.10.024).

42. Goacher, R. E.; Difonzo, L. G.; Lesko, K. C. Anal. Chem., 2017, 89, pp 759-766 (https://doi.org/10.1021/ acs.analchem.6b03411).

43. Martins, A. R.; Dourado, C. S.; Talhavini, M.; Braz, A.; Braga, J. W. B. Forensic Sci. Int., 2019, 296, pp 91-100 (https://doi.org/10.1016/j.forsciint.2019.01.021).

44. Rodrigues e Brito, L.; Chaves, A. B.; Braz, A.; Pimentel, M. F. Spectrochim. Acta, Part A, 2019, 223, 117287 (https://doi.org/10.1016/j.saa.2019.117287).

45. Rodrigues e Brito, L.; Braz, A.; Honorato, R. S.; Pimentel, M. F.; Pasquini, C. Forensic Sci. Int., 2019, 298, pp 169-176 (https://doi.org/10.1016/j.forsciint.2019.02.043).

46. Ramli, S.; Talib, R. A.; Rahman, R. A.; Zainuddin, N.; Othman, S. H.; Rashid, N. M. J. Spectrosc., 2015 (https://doi.org/10.1155/2015/502340).

47. Haase, E.; Arroyo, L.; Trejos, T. Spectrochim. Acta, Part B., 2020, 172 (https://doi.org/10.1016/j. sab.2020.105963).

48. Melendez-Perez, J. J.; Correa, D. N.; Hernandes, V. V.; De Morais, D. R.; De Oliveira, R. B.; De Souza, W. J.; Santos, M.; Eberlin, M. N. Appl. Spectrosc., 2016, 16, pp 1910-1915 (https://doi. org/10.1177/0003702816645352).

49. Hoehse, M.; Paul, A.; Gornushkin, I.; Panne, U. Anal. Bioanal. Chem., 2012, 402, pp 1443-1450 (https://doi.org/10.1007/s00216-011-5287-6).

50. Trejos, T.; Torrione, P.; Corzo, R.; Raeva, A.; Subedi, K.; Williamson, R.; Yoo, J.; Almirall, J. J. Forensic Sci., 2016, 61, pp 715-724 (https://doi.org/10.1111/1556-4029.13109).

51. Kumar, R.; Samkaria, A.; Sharma, V. Sci. Justice, 2020, 60, pp 347-357 (https://doi.org/10.1016/j. scijus.2020.01.004). 
52. Oravec, M.; Beganović, A.; Gál, L.; Čeppan, M.; Huck, C. W. Forensic Sci. Int., 2019, 299, pp 128-134 (https://doi.org/10.1016/j.forsciint.2019.03.041).

53. Materazzi, S.; Risoluti, R.; Pinci, S.; Romolo, F. S. Talanta, 2017, 174, pp 673-678 (https://doi. org/10.1016/j.talanta.2017.06.044).

54. Verma, N.; Sharma, V.; Kumar, R.; Sharma, R.; Joshi, M. C.; Umapathy, G. R.; Ohja, S.; Chopra, S. Anal. Bioanal. Chem., 2019, 411, pp 3477-3495 (https://doi.org/10.1007/s00216-019-01824-z).

55. Verma, N.; Kumar, R.; Sharma, V. Spectrochim. Acta - Part A, 2018, 196, pp $40-48$ (https://doi. org/10.1016/j.saa.2018.02.001).

56. Gál, L.; Oravec, M.; Kiššová, M.; Gemeiner, P.; Čeppan, M. Chem. Pap., 2020, 74, pp 3269-3277 (https://doi.org/10.1007/s11696-020-01145-x).

57. Farid, S.; Kasem, M. A.; Zedan, A. F.; Mohamed, G. G.; El-Hussein, A. Opt. Laser Technol., 2021, 135 (https://doi.org/10.1016/j.optlastec.2020.106704).

58. Sugawara, S.; Huck, C. W. Infrared Phys. Technol., 2020, 105 (https://doi.org/10.1016/j. infrared.2020.103212).

59. Valderrama, L.; Março, P. H.; Valderrama, P. J. Chemom., 2020, 34 (12), e3265 (https://doi.org/10.1002/ cem.3265).

60. Xia, J.; Zhang, J.; Zhao, Y.; Huang, Y.; Xiong, Y.; Min, S. Spectrochim. Acta - Part A, 2019, 219, pp 8-14 (https://doi.org/10.1016/j.saa.2018.09.059).

61. Kumar, R.; Sharma, V.; Verma, N.; Diwan, P. K.; Kumar, V.; Kumar, V. Aust. J. Forensic Sci., 2019, 51, pp 22-39 (https://doi.org/10.1080/00450618.2017.1310921).

62. Kumar, R.; Kumar, V.; Sharma, V. Spectrochim. Acta - Part A, 2017, 170, pp 19-28 (https://doi. org/10.1016/j.saa.2016.06.042).

63. Xia, J.; Huang, Y.; Zhang, J.; Du, X.; Yan, H.; Li, Q.; Li, Y.; Xiong, Y.; Min, S. Cellulose, 2020, 27, pp 5323-5335 (https://doi.org/10.1007/s10570-019-02892-1).

64. Ortiz-Herrero, L.; Blanco, M. E.; García-Ruiz, C.; Bartolomé, L. J. Anal. Appl. Pyrolysis, 2018, 131, pp 9-16 (https://doi.org/10.1016/j.jaap.2018.02.018).

65. Silva, C. S.; Pimentel, M. F.; Amigo, J. M.; García-Ruiz, C.; Ortega-Ojeda, F. Anal. Chim. Acta, 2018, 1031, pp 28-37 (https://doi.org/10.1016/j.aca.2018.06.031).

66. Sharma, V.; Kaur, J.; Kumar, R. Aust. J. Forensic Sci., 2020 (https://doi.org/10.1080/00450618.2020. 1781254).

67. Trafela, T.; Mizuno, M.; Fukunaga, K.; Strlič, M. THz spectroscopy and chemometrics for quantitative determination of chemical properties and dating of historic paper. $35^{\text {th }}$ International Conference on Infrared, Millimeter, and Terahertz Waves, 2010 (https://doi.org/10.1109/ICIMW.2010.5612350).

68. Vittorazzi, B. V.; Costa, R. A.; Coelho, L. M.; Isidoro, M. M.; Lima, K. M. G.; Filgueiras, P. R.; Romão, W. Quim. Nova, 2020, 43, pp 447-454 (https://doi.org/10.21577/0100-4042.20170508).

69. Rodrigues, A. R. N.; Melquiades, F. L.; Appoloni, C. R.; Marques, E. N. Forensic Sci. Int., 2019, 302, 109872 (https://doi.org/10.1016/j.forsciint.2019.06.030).

70. Appoloni, C. R.; Melquiades, F. L. Appl. Radiat. Isot., 2014, 85, pp 92-95 (https://doi.org/10.1016/j. apradiso.2013.12.004).

71. de Almeida, M. R.; Correa, D. N.; Rocha, W. F. C.; Scafi, F. J. O.; Poppi, R. J. Microchem. J., 2013, 109, pp 170-177 (https://doi.org/10.1016/j.microc.2012.03.006).

72. Gong, F.; Wang, B. T.; Chau, F. T.; Liang, Y. Z. Anal. Lett., 2005, 38, pp 2475-2492 (https://doi. org/10.1080/00032710500318338).

73. Geladi, P. Spectrochim. Acta - Part B, 2003, 58, pp 767-782 (https://doi.org/10.1016/S05848547(03)00037-5).

74. Gadžurić, S. B.; Podunavac, S. O.; Kuzmanović, M. B.; Vraneš, M.; Petrin, T.; Bugarski, S. Z.; Kovačević, S. Z. Iran. J. Pharm. Res., 2016, 15, pp 725-734 (https://doi.org/10.22037/ijpr.2016.1905).

75. Sarker, S. D.; Nahar, L. Applications of High Performance Liquid Chromatography in the Analysis of Herbal Products. In: Mukherjee, P. K. (Ed.) Evidence-Based Validation of Herbal Medicine. Elsevier Inc., 2015, Chapter 19, pp 405-425 (https://doi.org/10.1016/B978-0-12-800874-4.00019-2). 
76. Santana, F. B.; Souza, A. M.; Almeida, M. R.; Breitkreitz, M. C.; Filgueiras, P. R.; Sena, M. M.; Poppi, R. J. Quim. Nova, 2020, 43, pp 371-381 (https://doi.org/10.21577/0100-4042.20170480).

77. Silva, C. S.; Braz, A.; Pimentel, M. F. J. Braz. Chem. Soc., 2019, 30, pp 2259-2290 (https://doi. org/10.21577/0103-5053.20190140).

78. Duarte, A. C.; Capelo, S. J. Liq. Chromatogr. Relat. Technol., 2006, 29, pp 1143-1176 (https://doi. org/10.1080/10826070600574929).

79. De Lathauwer, L.; De Moor, B.; Vandewalle, J. J. Chemom., 2000, 14, pp 123-149 (https://doi. org/10.1002/1099-128X(200005/06)14:3<123::AID-CEM589>3.0.CO;2-1).

80. Noble, W. S. Nat. Biotechnol., 2006, 24, pp 1565-1567 (https://doi.org/10.1038/nbt1206-1565).

81. Março, P. H.; Valderrama, P.; Alexandrino, G. L.; Poppi, R. J.; Tauler, R. Quim. Nova., 2014, 37, pp 1525-1532 (https://doi.org/10.5935/0100-4042.20140205). 